\section{BRAZIULIAN JOURNAL \\ OF MEDICAL AND BIOLOGICAL RESF.ARCH}

www.bjournal.com.br
ISSN 0100-879X

Volume 42 (11) 993-1118 November 2009

BIOMEDICAL SCIENCES

AND

CLINICAL INVESTIGATION

Braz J Med Biol Res, November 2009, Volume 42(11)1044-1049

Stem cell factor protects against neuronal apoptosis by activating AKT/ERK in diabetic mice

J.-W. Li, L.-L. Li, L.-L. Chang, Z.-Y. Wang and Y. Xu

The Brazilian Journal of Medical and Biological Research is partially financed by
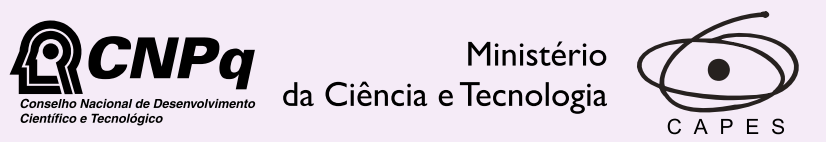

Ministério da Educação

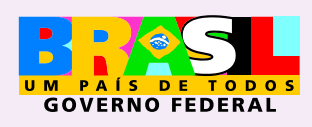

DFAPESP

Institutional Sponsors 


\title{
Stem cell factor protects against neuronal apoptosis by activating AKT/ERK in diabetic mice
}

\author{
J.-W. Li $i^{1,3,4 *}$, L.-L. Li ${ }^{2 *}$, L.-L. Chang ${ }^{1}$, Z.-Y. Wang ${ }^{3,4,5}$ and Y. Xu1,2,3,4,5 \\ ${ }^{1}$ Department of Neurology, Affiliated Drum Tower Hospital, Nanjing Medical University, Nanjing, Jiangsu, China \\ 2Department of Neurology, Affiliated Drum Tower Hospital of Nanjing, School of Clinical Medicine, \\ Southeast University, China \\ ${ }^{3}$ Department of Neurology, Affiliated Drum Tower Hospital, Nanjing University Medical School, Nanjing, \\ Jiangsu, China \\ ${ }^{4}$ Jiangsu Key Laboratory for Molecular Medicine, Nanjing, Jiangsu, China \\ ${ }^{5}$ The State Key Laboratory of Pharmaceutical Biotechnology, Nanjing University, \\ Nanjing, Jiangsu, China
}

\begin{abstract}
Neuronal apoptosis occurs in the diabetic brain due to insulin deficiency or insulin resistance, both of which reduce the expression of stem cell factor (SCF). We investigated the possible involvement of the activation of the MAPK/ERK and/or AKT pathways in neuroprotection by SCF in diabetes. Male C57/B6 mice (20-25 g) were randomly divided into four groups of 10 animals each. The morphology of the diabetic brain in mice treated or not with insulin or SCF was evaluated by H\&E staining and TUNEL. SCF, ERK1/2 and AKT were measured by Western blotting. In diabetic mice treated with insulin or SCF, there was fewer structural change and apoptosis in the cortex compared to untreated mice. The apoptosis rate of the normal group, the diabetic group receiving vehicle, the diabetic group treated with insulin, and the diabetic group treated with SCF was 0.54 $\pm 0.077 \%, 2.83 \pm 0.156 \%, 1.86 \pm 0.094 \%$, and $1.78 \pm 0.095 \%$ (mean \pm SEM), respectively. SCF expression was lower in the diabetic cortex than in the normal cortex; however, insulin increased the expression of SCF in the diabetic cortex. Furthermore, expression of phosphorylated ERK1/2 and AKT was decreased in the diabetic cortex compared to the normal cortex. However, insulin or SCF could activate the phosphorylation of ERK1/2 and AKT in the diabetic cortex. The results suggest that SCF may protect the brain from apoptosis in diabetes and that the mechanism of this protection may, at least in part, involve activation of the ERK1/2 and AKT pathways. These results provide insight into the mechanisms by which SCF and insulin exert their neuroprotective effects in the diabetic brain.
\end{abstract}

Key words: Stem cell factor; Diabetes; MAPK/ERK; AKT

\section{Introduction}

Although structural abnormalities in neural tissue such as synaptic and neuronal degeneration and neuronal apoptosis have been demonstrated in type 1 and type 2 diabetic rodents (1-3), very little is known about the mechanisms leading to these defects. Many researchers have suggested that neuronal apoptosis in diabetes is related to hyperglycemia (4), and insulin, proinsulin $C$ peptide and insulin-like growth factor (IGF) have anti-apoptotic effects $(5,6)$. Furthermore, insulin or insulin-like growth factor-1 (IGF-1) induced the expression of stem cell factor (SCF) and a lower expression of SCF was observed in the mouse gut during diabetic gastroparesis $(7,8)$. SCF, also termed Kit ligand, steel factor, mast cell growth factor, or c-kit ligand, is a receptor of tyrosine kinase. Both SCF and Kit support neuronal survival and function and influence cell differentiation, migration, and proliferation possibly by mutual interaction $(9,10)$. Knockdown of SCF results in abnormalities in learning and memory in mice $(11,12)$. SCF is a cytokine highly expressed in the central nervous system and protects neurons from apoptosis $(13,14)$. SCF-mediated signal

Correspondence: Y. Xu, Department of Neurology, Affiliated Drum Tower Hospital, Nanjing University Medical School, 321 ZhongShan Road, Nanjing, Jiangsu, 210008, China. Fax: +86-25-8331-7016. E-mail: xuyun20042001@yahoo.com.cn

Research supported by the National Nature Science Foundation (\#30470612, \#30670739) of China, the Doctoral Program Foundation of the Ministry of Education of China (\#20060284044), and the International Cooperation Program and Outstanding Researcher Program (\#BZ2006045, \#06-B-002, \#RC2007006) of Jiangsu Province of China.

*These authors contributed equally to this study.

Received December 27, 2008. Accepted August 24, 2009. Available online October 5, 2009. 
transduction may involve many signaling pathways. The cytoprotection induced by SCF is associated with activation of the AKT and/or MAPK signaling pathways $(14,15)$. In the present study, we tested the hypothesis that SCF protects neurons from apoptosis in diabetes via a mechanism that partially involves the AKT and ERK pathways.

\section{Material and Methods}

\section{Animal model}

The animal study was conducted in accordance with National Regulations of Experimental Animal Administration. All experimental animal protocols were approved by the Committee of Experimental Animal Administration of Nanjing Medical University. Diabetes was induced in male C57/B6 mice (20-25 g) with a single intraperitoneal injection of streptozotocin $(100 \mathrm{mg} / \mathrm{kg}$ in $0.1 \mathrm{M}$ citrate buffer, $\mathrm{pH} 4.4$; Sigma, USA); $72 \mathrm{~h}$ later the plasma glucose level of the mice (diabetes type I model) was above $300 \mathrm{mg} /$ $\mathrm{dL}$ (OneTouch ${ }^{\circledR}$ Ultra Blood Glucose Monitoring System, Johnson, USA) (16). Plasma glucose was evaluated weekly for 4 weeks (see plasma glucose level in Figure 1). Control mice received an equal volume of citrate buffer. Mice were randomly divided into four groups of 10 animals each, i.e., normal control (CON), diabetic receiving vehicle (DM), diabetic treated with insulin (DM + INS: $40 \mathrm{U} \cdot \mathrm{kg}^{-1} \cdot \mathrm{day}^{-1}$; Wanbang Bio-pharm, China), and diabetic treated with SCF (DM + SCF: $0.1 \mathrm{mg} \cdot \mathrm{kg}^{-1} \cdot 48 \mathrm{~h}^{-1}$; BD, USA). Four weeks later seven brains from each group were rapidly removed and the cerebral cortices and hippocampi were isolated. Three brains from each group were transcardially perfused with cold $0.1 \mathrm{M}$ phosphate buffer, $\mathrm{pH} 7.4$, followed by $4.0 \%$ paraformaldehyde. The samples were stored at $-80^{\circ} \mathrm{C}$ for later experiments.

\section{HE staining and TUNEL assay}

Brain sections were stained with hematoxylin and eosin

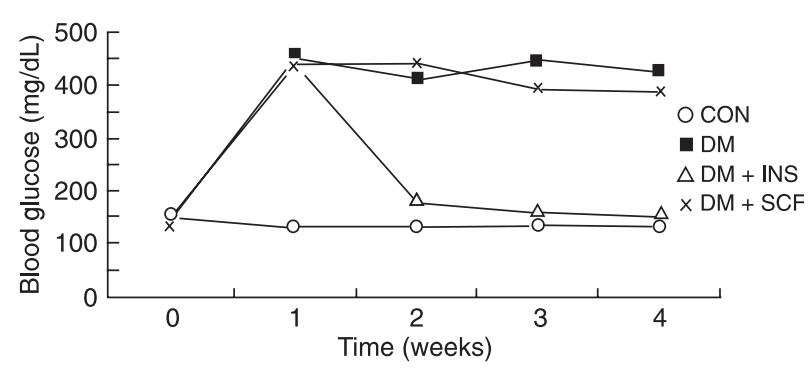

Figure 1. Plasma glucose curve. Type 1 diabetes was induced in mice by a single intraperitoneal injection of streptozotocin (100 $\mathrm{mg} / \mathrm{kg}$ ), with plasma glucose levels exceeding $250 \mathrm{mg} / \mathrm{dL} 72 \mathrm{~h}$ later. Plasma glucose was then measured weekly. $\mathrm{CON}=$ normal mice injected with citrate buffer; DM = diabetic mice injected with vehicle; $\mathrm{DM}+\mathrm{INS}=$ diabetic mice treated with insulin $(40 \mathrm{U}$. $\left.\mathrm{kg}^{-1} \cdot \mathrm{day}^{-1}\right) ; \mathrm{DM}+\mathrm{SCF}=$ diabetic mice treated with stem cell factor $\left(0.1 \mathrm{mg} \cdot \mathrm{kg}^{-1} \cdot 48 \mathrm{~h}^{-1}\right)$. for histological assay. Cell damage was also assessed by TUNEL (Boster, Wuhan, China), which was performed as previously described (17). Briefly, 20- $\mu$ m brain sections were incubated with TdT labeling buffer containing dUTP mix, $\mathrm{Mn}^{2+}$ and $\mathrm{TdT}$ enzyme for $60 \mathrm{~min}$ at $37^{\circ} \mathrm{C}$ to incorporate biotinylated nucleotides at the sites of DNA breaks. Sections were also incubated with diluted extraavidin-peroxidase link for $30 \mathrm{~min}$ at $37^{\circ} \mathrm{C}$, followed by the addition of $D A B$ (diaminobenzidine tetrahydrochloride) at room temperature to develop a brown color. They were counterstained with modified Harris' hematoxylin, changing to blue with TBS and observed microscopically. The number of cells exhibiting a positive TUNEL signal was quantified with the Soft Imaging System (Image Pro Plus 5.02, Media Cybernetics, Inc., USA).

\section{Western blotting}

Total protein and protein expression by the cortex was assayed as described previously (17). Membranes were probed with primary antibodies against SCF (1:500, BD), c-kit (1:500, Santa Cruz Biotechnology, USA), phosphorated ERK1/2, AKT (cell signaling, 1:1000), total ERK1/2, AKT or GAPDH (cell signaling, 1:1000). Proteins were detected using horseradish peroxidase-conjugated anti-goat or antirabbit secondary antibodies and visualized by chemiluminescence using an ECL kit (Amersham Pharmacia Biotech, USA) and exposure to film. The intensity of the blots was quantified by densitometry (AlphaEaseFC, Alpha Innotech Corporation, USA). GAPDH, total ERK or total AKT were used as loading controls.

\section{Statistical analysis}

Data are reported as means \pm SEM. One-way ANOVA followed by multiple comparisons was used for statistical comparisons. In all statistical procedures, a probability value of $P<0.05$ was used as a cutoff for statistical significance.

\section{Results and Discussion}

\section{Cortical apoptosis appeared in the diabetic mice}

Model type 1 diabetic mice (blood glucose level 300-450 $\mathrm{mg} / \mathrm{dL}$ ) were prepared using streptozotocin. To confirm previous studies, pathology and cell apoptosis were determined in the diabetic brains. Histological results confirmed strong staining of the nuclei, an intensely eosinophilic cytoplasm and widening of Virchow-Robin spaces. Along with these changes, neuronal apoptosis occurred in the same areas (Figure 2B,C). CON, DM + SCF or INS animals showed few pathological changes of this kind (Figure 2A). The pathological injuries in the cortex of diabetic mice, including structural changes and apoptosis, were fewer in animals pretreated with insulin or SCF. However, in contrast to previous studies, the hippocampus did not show structural changes or apoptosis (Figure 3A,B). In other words, there 
is a diabetes-related programmed neuronal cell death in the hippocampus, which results in neuronal loss in type 1 diabetes $(18,19)$. Different results might be obtained using different models or at different times after experimental hyperglycemia. To test this model, we used a lower dose of streptozotocin and performed the experiment by the 4th week. The observed difference in structure changes may have been related to milder diabetic damage.

\section{SCF protects against apoptosis in the diabetic brain}

There is evidence that SCF expression levels can influence neuronal apoptosis in the diabetic brain. It is well known that diabetes results from insulin deficiency or variable degrees of insulin resistance. However, insulin or IGF-1 is a critical factor for inducing SCF expression and maintaining SCF signaling $(7,8)$. Reduced SCF resulted in smooth muscle myopathy and loss of interstitial cells of Cajal in murine diabetic gastroparesis (8). SCF and its receptor (c-kit) are expressed throughout the adult nervous system. SCF includes both membrane-bound SCF (M-SCF) and soluble SCF (S-SCF), the latter consisting of cleaved soluble forms of SCF $(9,10)$. The present study showed that the levels of both M-SCF and S-SCF in the diabetic cortex were lower than that in the normal cortex (Figure 4) and that insulin can increase both M-SCF and S-SCF in the diabetic brain. Further study revealed that cortical apoptosis appeared in the diabetic mice and that SCF could attenuate it (Figure 2).

In an increasing number of studies, it has been shown that neuronal expression of SCF is induced in various forms of brain injury and that SCF expression after brain injury is correlated with neurogenesis and the mechanism of brain

A
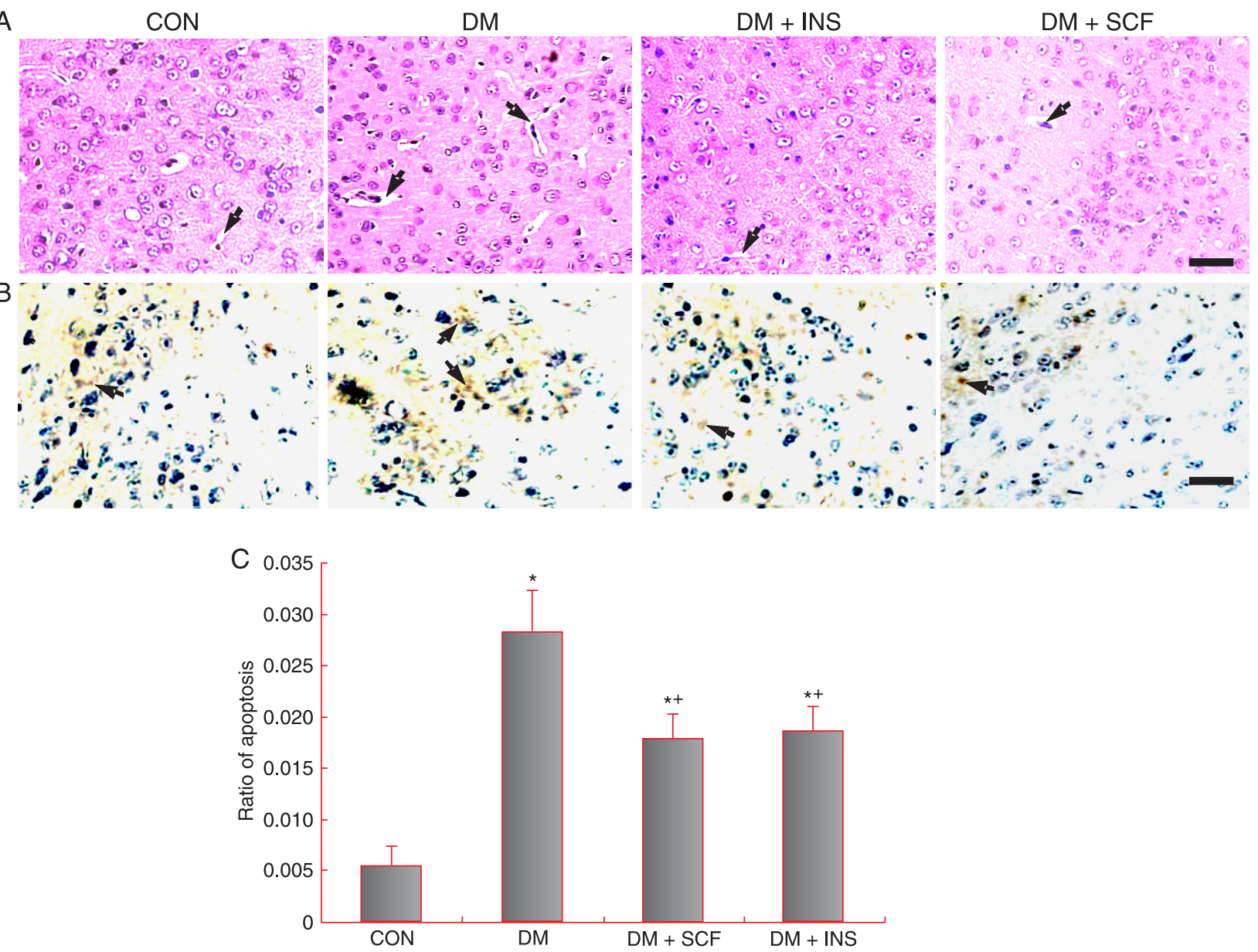

Figure 2. Morphology of the cortex. A, Hematoxylin and eosin staining. The DM group exhibited intensely eosinophilic cytoplasm, condensed nuclei, and widening of Virchow-Robin spaces (arrows). CON, DM + SCF, or DM + INS showed few of these pathological changes. Bar $=25 \mu \mathrm{m} . B$, TUNEL assay. Many apoptotic cells (arrows, brown color) appeared in the DM group, and less apoptosis occurred in DM + SCF, and DM + INS groups. Bar $=40 \mu \mathrm{m}$. C, Quantitation of TUNEL assay. The apoptosis rates of CON, DM, DM + INS, and DM + SCF groups were $0.54 \pm 0.077 \%, 2.83 \pm 0.156 \%, 1.86 \pm 0.094 \%$, and $1.78 \pm 0.095 \%$, respectively. ${ }^{*} \mathrm{P}<0.05$ compared to CON group, ${ }^{+}$compared to DM group (one-way ANOVA). For abbreviations, see legend to Figure 1. 
A

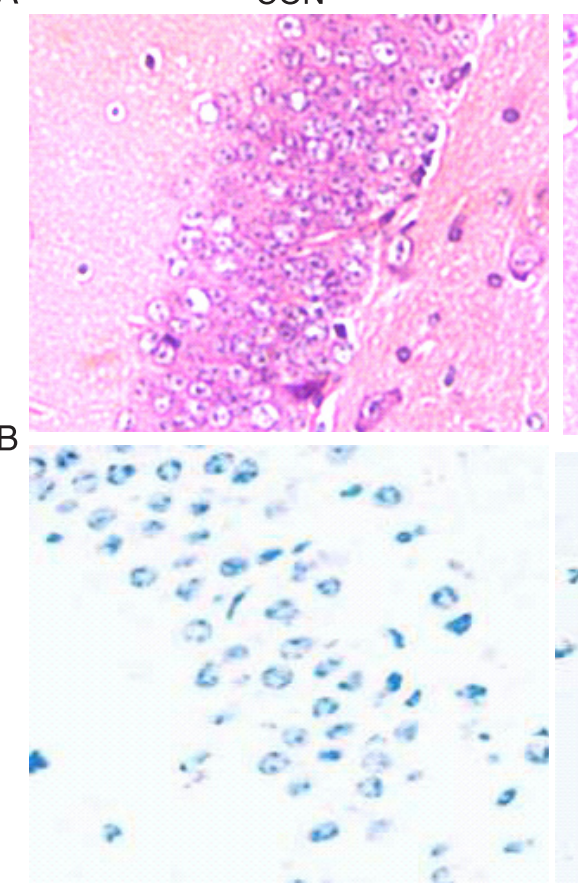

DM

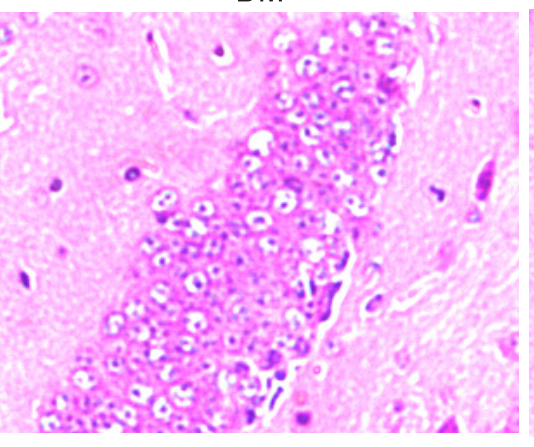

$D M+I N S$

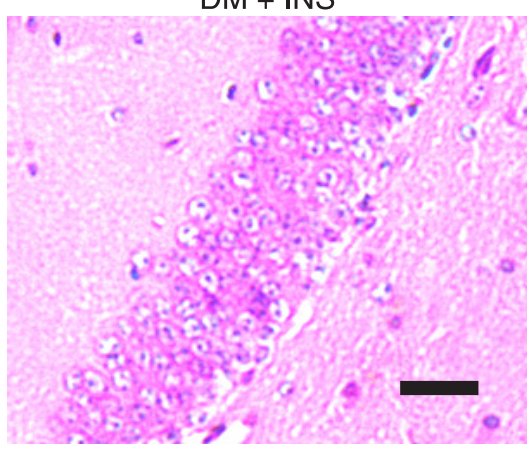

Figure 3. Morphology of the hippocampus. A, Hematoxylin and eosin staining. No significant structure change among the three groups was found in the hippocampus. Bar $=40 \mu \mathrm{m} . B$, TUNEL assay. No apoptosis was observed in the hippocampus among the three groups. Bar $=25 \mu \mathrm{m}$. For abbreviations, see legend to Figure 1.

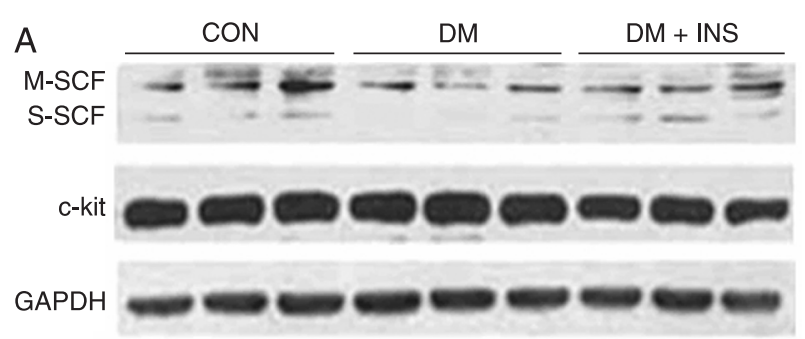

$\mathrm{B}$

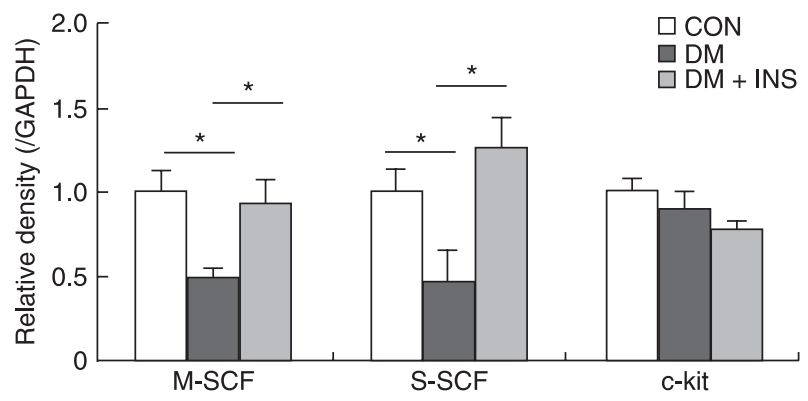

Figure 4. $A$, Expression of M-SCF, S-SCF and c-kit by Western blotting. $B$, Relative density of $A$. Expression of SCF (membranebound SCF, M-SCF and soluble SCF, S-SCF) in the DM group was lower than in the CON group; DM + INS increased the expression of SCF. There were no significant differences among the three groups in the expression of c-kit. ${ }^{*} \mathrm{P}<0.05$ (one-way ANOVA). For abbreviations, see legend to Figure 1. repair (14,20-22). SCF protects against camptothecin and glutamate-induced neuronal cell death $(13,14)$. SCF and Kit are responsible for the survival of a variety of cells including neuronal cells and hematopoietic lineages (23). Targeted disruption of the kit gene leads to marked defects in learning and memory (12), also a key manifestation of diabetic encephalopathy. In the present study, no abnormal expression of c-kit was found in the diabetic brain. Kit alteration may possibly follow alterations in SCF. Our model using milder diabetic damage suggests that pathological injury in the diabetic brain may first occur in the cortex and only later extend to the hippocampus. Our model further suggests that SCF/c-kit expression may be used to evaluate the severity of diabetic encephalopathy.

\section{Neuroprotection in diabetes by SCF via activation of AKT/ERK}

To further investigate which signaling pathway is involved in neuroprotection by SCF in diabetes, diabetic mice were pretreated with insulin, SCF or vehicle and the subsequent activation of MAPK/ERK1/2 and AKT in the cortex was determined by Western blotting, as described in Figure 5. The results indicated that there was less activation of ERK $1 / 2$ and AKT in the cortex of the diabetic brain than in the normal cortex. However, the addition of SCF or insulin to this model effectively activated MAPK and AKT. Both the MAPK/ERK and PI-3K/Akt signaling pathways are essential 
A

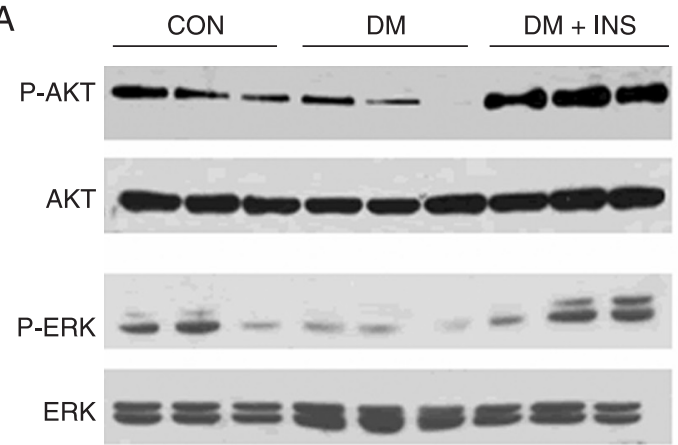

B

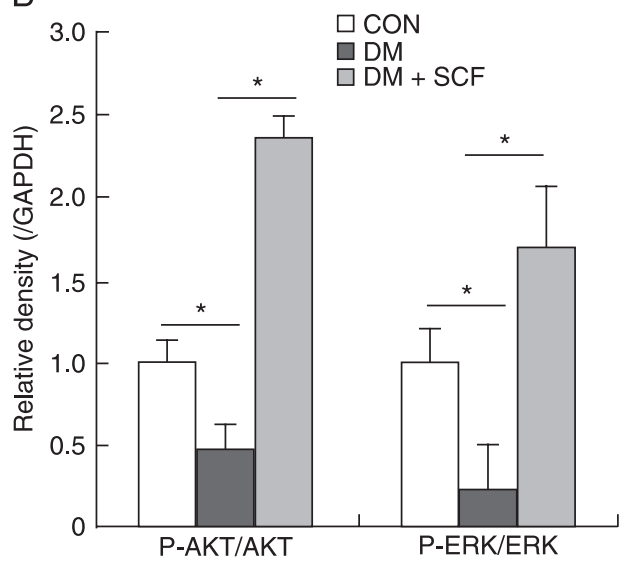

C
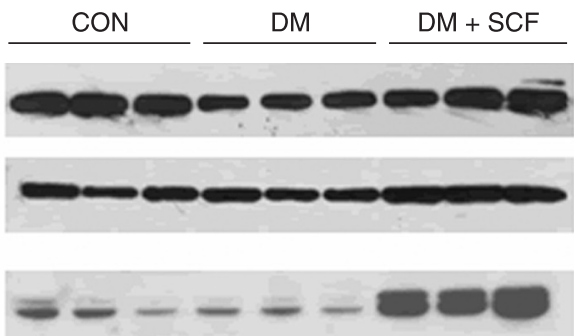

$z=\mathrm{z}=\mathrm{z}=\mathrm{z}$

D

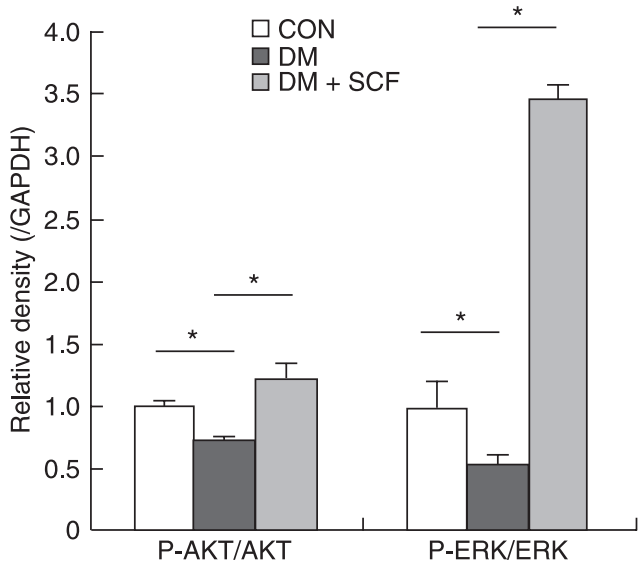

Figure 5. $A, C$, Expression of phosphorylated AKT (P-AKT) and ERK (P-ERK) by Western blotting. $B, D$, Relative density of $A$ and $C$. There is decreased activation of AKT and ERK in DM; INS or SCF increased the expression of phosphorylated AKT and ERK. *P $<$ 0.01 (one-way ANOVA). For abbreviations, see legend to Figure 1.

in the prevention of neuronal apoptosis and are also implicated in the protection of the brain from ischemic stroke and Alzheimer's disease $(14,17)$. SCF also suppressed camptothecin and glutamate-induced neuronal apoptosis via MAPK/ERK and PI-3K/Akt activation. SCF-mediated signal transduction may involve a different cell signaling pathway (14). Interestingly, SCF-mediated cytoprotection in non-central nervous system cell types is also linked to activation of the AKT and/or the MAPK signaling pathways $(24,25)$, suggesting that a similar mechanism of action of SCF/c-kit may be involved in neuroprotection and in the protection of other cell types.

\section{References}

1. Bestetti G, Rossi GL. Hypothalamic lesions in rats with long-term streptozotocin-induced diabetes mellitus. A semiquantitative light- and electron-microscopic study. Acta Neuropathol 1980; 52: 119-127.

2. Mukai N, Hori S, Pomeroy M. Cerebral lesions in rats with
Cortical neuronal apoptosis appeared in the diabetic brain due to reduced SCF expression and this apoptosis could be inhibited by SCF. The mechanism by which this occurred may be correlated to the activation of the ERK and AKT pathways. Accordingly, SCF may hold promise for development as a neuroprotectant for treating diabetesrelated brain injury.

\section{Acknowledgments}

We thank Mrs. Marilyn White for editing the manuscript.

streptozotocin-induced diabetes. Acta Neuropathol 1980; 51: 79-84.

3. Mijnhout GS, Scheltens P, Diamant M, Biessels GJ, Wessels AM, Simsek S, et al. Diabetic encephalopathy: A concept in need of a definition. Diabetologia 2006; 49: 1447-1448. 
4. Srinivasan S, Stevens M, Wiley JW. Diabetic peripheral neuropathy: evidence for apoptosis and associated mitochondrial dysfunction. Diabetes 2000; 49: 1932-1938.

5. Li ZG, Zhang W, Sima AA. C-peptide enhances insulinmediated cell growth and protection against high glucoseinduced apoptosis in SH-SY5Y cells. Diabetes Metab Res Rev 2003; 19: 375-385.

6. Kuemmerle JF. Endogenous IGF-I protects human intestinal smooth muscle cells from apoptosis by regulation of GSK-3 beta activity. Am J Physiol Gastrointest Liver Physiol 2005; 288: G101-G110.

7. Hsueh WA, Law RE. Insulin signaling in the arterial wall. Am J Cardiol 1999; 84: 21J-24J.

8. Horvath VJ, Vittal H, Lorincz A, Chen H, Almeida-Porada G, Redelman $D$, et al. Reduced stem cell factor links smooth myopathy and loss of interstitial cells of Cajal in murine diabetic gastroparesis. Gastroenterology 2006; 130: 759-770.

9. Ashman LK. The biology of stem cell factor and its receptor C-kit. Int J Biochem Cell Biol 1999; 31: 1037-1051.

10. Hollenbeck ST, Sakakibara K, Faries PL, Workhu B, Liu B, Kent KC. Stem cell factor and c-kit are expressed by and may affect vascular SMCs through an autocrine pathway. $J$ Surg Res 2004; 120: 288-294.

11. Motro B, Wojtowicz JM, Bernstein A, van der Kooy D. Steel mutant mice are deficient in hippocampal learning but not long-term potentiation. Proc Natl Acad Sci U S A 1996; 93: 1808-1813.

12. Katafuchi T, Li AJ, Hirota S, Kitamura Y, Hori T. Impairment of spatial learning and hippocampal synaptic potentiation in c-kit mutant rats. Learn Mem 2000; 7: 383-392.

13. Manova K, Bachvarova RF, Huang EJ, Sanchez S, Pronovost SM, Velazquez E, et al. c-kit receptor and ligand expression in postnatal development of the mouse cerebellum suggests a function for c-kit in inhibitory interneurons. $J$ Neurosci 1992; 12: 4663-4676.

14. Dhandapani KM, Wade FM, Wakade C, Mahesh VB, Brann DW. Neuroprotection by stem cell factor in rat cortical neurons involves AKT and NFkappaB. J Neurochem 2005; 95: 9-19.

15. Matsui J, Wakabayashi T, Asada M, Yoshimatsu K, Okada M.
Stem cell factor/c-kit signaling promotes the survival, migration, and capillary tube formation of human umbilical vein endothelial cells. J Biol Chem 2004; 279: 18600-18607.

16. Biessels GJ, Gispen WH. The impact of diabetes on cognition: what can be learned from rodent models? Neurobiol Aging 2005; 26 (Suppl 1): 36-41.

17. Xu Y, Zhang W, Klaus J, Young J, Koerner I, Sheldahl LC, et al. Role of cocaine- and amphetamine-regulated transcript in estradiol-mediated neuroprotection. Proc Natl Acad Sci U $S$ A 2006; 103: 14489-14494.

18. Li ZG, Zhang W, Grunberger G, Sima AA. Hippocampal neuronal apoptosis in type 1 diabetes. Brain Res 2002; 946: 221-231.

19. Sima AA, Li ZG. The effect of C-peptide on cognitive dysfunction and hippocampal apoptosis in type 1 diabetic rats. Diabetes 2005; 54: 1497-1505.

20. Kelly DJ, Zhang Y, Gow RM, Itescu S, Gilbert RE. Cells expressing the stem cell factor receptor, c-kit, contribute to neoangiogenesis in diabetes. Diab Vasc Dis Res 2005; 2: 76-80.

21. Jin K, Mao XO, Sun Y, Xie L, Greenberg DA. Stem cell factor stimulates neurogenesis in vitro and in vivo. $J$ Clin Invest 2002; 110: 311-319.

22. Sun L, Lee J, Fine HA. Neuronally expressed stem cell factor induces neural stem cell migration to areas of brain injury. $J$ Clin Invest 2004; 113: 1364-1374.

23. Gore BB, Wong KG, Tessier-Lavigne M. Stem cell factor functions as an outgrowth-promoting factor to enable axon exit from the midline intermediate target. Neuron 2008; 57 : 501-510.

24. Edling CE, Pedersen M, Carlsson L, Ronnstrand L, Palmer $\mathrm{RH}$, Hallberg B. Haematopoietic progenitor cells utilise conventional PKC to suppress PKB/Akt activity in response to c-Kit stimulation. Br J Haematol 2007; 136: 260-268.

25. Kapur R, Chandra S, Cooper R, McCarthy J, Williams DA. Role of p38 and ERK MAP kinase in proliferation of erythroid progenitors in response to stimulation by soluble and membrane isoforms of stem cell factor. Blood 2002; 100: 1287-1293. 